\title{
Fast and Powerful Hashing using Tabulation
}

\author{
Mikkel Thorup* \\ University of Copenhagen
}

January 4, 2017

\begin{abstract}
Randomized algorithms are often enjoyed for their simplicity, but the hash functions employed to yield the desired probabilistic guarantees are often too complicated to be practical. Here we survey recent results on how simple hashing schemes based on tabulation provide unexpectedly strong guarantees.

Simple tabulation hashing dates back to Zobrist [1970]. Keys are viewed as consisting of $c$ characters and we have precomputed character tables $h_{1}, \ldots, h_{c}$ mapping characters to random hash values. A key $x=\left(x_{1}, \ldots, x_{c}\right)$ is hashed to $h_{1}\left[x_{1}\right] \oplus h_{2}\left[x_{2}\right] \ldots . \oplus h_{c}\left[x_{c}\right]$. This schemes is very fast with character tables in cache. While simple tabulation is not even 4-independent, it does provide many of the guarantees that are normally obtained via higher independence, e.g., linear probing and Cuckoo hashing.

Next we consider twisted tabulation where one input character is "twisted" in a simple way. The resulting hash function has powerful distributional properties: Chernoff-style tail bounds and a very small bias for min-wise hashing. This also yields an extremely fast pseudo-random number generator that is provably good for many classic randomized algorithms and data-structures.

Finally, we consider double tabulation where we compose two simple tabulation functions, applying one to the output of the other, and show that this yields very high independence in the classic framework of Carter and Wegman [1977]. In fact, w.h.p., for a given set of size proportional to that of the space consumed, double tabulation gives fully-random hashing. We also mention some more elaborate tabulation schemes getting near-optimal independence for given time and space.

While these tabulation schemes are all easy to implement and use, their analysis is not.
\end{abstract}

\section{Introduction}

A useful assumption in the design of randomized algorithms and data structures is the free availability of fully random hash functions which can be computed in unit time. Removing this unrealistic assumption is the subject of a large body of work. To implement a hash-based algorithm, a concrete hash function has to be chosen. The space, time, and random choices made by this hash function affects the overall performance. The generic goal is therefore to provide efficient constructions of hash functions that for important randomized algorithms yield probabilistic guarantees similar to those obtained assuming fully random hashing.

To fully appreciate the significance of this program, we note that many randomized algorithms are very simple and popular in practice, but often they are implemented with too simple hash functions without the necessary guarantees. This may work very well in random tests, adding to their popularity, but the real world

\footnotetext{
${ }^{*}$ Research partly supported by Advanced Grant DFF-0602-02499B from the Danish Council for Independent Research under the Sapere Aude research carrier programme.
} 
is full of structured data, e.g., generated by computers, that could be bad for the hash function. This was illustrated in [51] showing how simple common inputs made linear probing fail with popular hash functions, explaining its perceived unreliability in practice. The problems disappeared when sufficiently strong hash functions were used.

In this paper we will survey recent results from $[12,13,14,15,41,42,50]$ showing how simple realistic hashing schemes based on tabulation provide unexpectedly strong guarantees for many popular randomized algorithms, e.g., linear probing, Cuckoo hashing, min-wise independence, treaps, planar partitions, powerof-two-choices, Chernoff-style concentration bounds, and even high independence. The survey is from a users perspective, explaining how these tabulation schemes can be applied. While these schemes are all very simple to describe and use, the analysis showing that they work is non-trivial. For the analysis, the reader will be referred to the above papers. The reader is also referred to these papers for a historical account of previous work.

Background Generally a hash function maps a key universe $\mathcal{U}$ of keys into some range $\mathcal{R}$ of hash values. A random hash function $h$ is a random variable from $\mathcal{R}^{\mathcal{U}}$, assigning a random hash value $h(x) \in \mathcal{R}$ to every $x \in \mathcal{U}$. A truly random hash function is picked uniformly from $\mathcal{R}^{\mathcal{U}}$, assigning a uniform and independent hash value $h(x) \in \mathcal{R}$ to each key $x \in \mathcal{U}$. Often randomized algorithms are analyzed assuming access to truly random hash functions. However, just storing a truly random hash function requires $|\mathcal{U}| \log _{2}|\mathcal{R}|$ bits, which is unrealistic for large key universes.

In general, the keys may originate come from a very large universe $\mathcal{U}$. However, often we are only interested in the performance on an unknown set $S \subseteq \mathcal{U}$ of up to $n$ keys. Then our first step is to do a universe reduction, mapping $\mathcal{U}$ randomly to "signatures" in $[u]=\{0,1, \ldots, u-1\}$, where $u=n^{O(1)}$, e.g., $u=n^{3}$, so that no two keys from $S$ are expected to get the same signature [9]. Below we generally assume that this universe reduction has been done, if needed, hence that we "only" need to deal with keys from the polynomial universe $[u]$.

The concept of $k$-independence was introduced by Wegman and Carter [52] in FOCS'79 and has been the cornerstone of our understanding of hash functions ever since. As above, we think of a hash function $h:[u] \rightarrow[m]$ as a random variable distributed over $[m]^{[u]}$. We say that $h$ is $k$-independent if (a) for any distinct keys $x_{1}, \ldots, x_{k} \in[u]$, the hash values $h\left(x_{1}\right), \ldots, h\left(x_{k}\right)$ are independent random variables; and (b) for any fixed $x, h(x)$ is uniformly distributed in $[m]$.

As the concept of independence is fundamental to probabilistic analysis, $k$-independent hash functions are both natural and powerful in algorithm analysis. They allow us to replace the heuristic assumption of truly random hash functions that are uniformly distributed in $[m]^{[u]}$, hence needing $u \lg m$ random bits $\left(\lg =\log _{2}\right)$, with real implementable hash functions that are still "independent enough" to yield provable performance guarantees similar to those proved with true randomness. We are then left with the natural goal of understanding the independence required by hashing-based algorithms.

Once we have proved that $k$-independence suffices for a hashing-based randomized algorithm, we are free to use any $k$-independent hash function. The canonical construction of a $k$-independent hash function is based on polynomials of degree $k-1$. Let $p \geq u$ be prime. Picking random $a_{0}, \ldots, a_{k-1} \in\{0, \ldots, p-1\}$, the hash function is defined by:

$$
h(x)=\left(\left(a_{k-1} x^{k-1}+\cdots+a_{1} x+a_{0}\right) \bmod p\right)
$$

If we want to limit the range of hash values to $[m]$, we use $h(x) \bmod m$. This preserves requirement (a) of independence among $k$ hash values. Requirement (b) of uniformity is close to satisfied if $p \gg m$. As 
suggested in [9], for a faster implementation, we can let $p$ be a Mersenne prime, e.g., to hash 64-bit integers, we could pick $p=2^{81}-1$.

Sometimes 2-independence suffices. For example, 2-independence implies so-called universality [9]; namely that the probability of two keys $x$ and $y$ colliding with $h(x)=h(y)$ is $1 / m$; or close to $1 / m$ if the uniformity of (b) is only approximate. Universality implies expected constant time performance of hash tables implemented with chaining. Universality also suffices for the 2-level hashing of Fredman et al. [24], yielding static hash tables with constant query time. Moreover, Mitzenmacher and Vadhan [34] have proved that 2-independent hashing in many applications works almost like truly random hashing if the input has enough entropy. However, structured, low-entropy data, are very common in the real world.

We do have very fast implementations of universal and 2-independent hashing [16, 17], but unfortunately, these methods do not generalize nicely to higher independence.

At the other end of the spectrum, when dealing with problems involving $n$ objects, $O(\lg n)$-independence suffices in a vast majority of applications. One reason for this is the Chernoff bounds of [43] for $k$ independent events, whose probability bounds differ from the full-independence Chernoff bound by $2^{-\Omega(k)}$. Another reason is that random graphs with $O(\lg n)$-independent edges [3] share many of the properties of truly random graphs.

When it comes to high independence, we note that the polynomial method from Equation (1) takes $O(k)$ time and space for $k$-independence. This is no coincidence. Siegel [46] has proved that to implement $k$ independence with less than $k$ memory accesses, we need a representation using $u^{1 / k}$ space. He also gives a solution that for any $c$ uses $O\left(u^{1 / c}\right)$ space, $c^{O(c)}$ evaluation time, and achieves $u^{\Omega\left(1 / c^{2}\right)}$ independence (which is superlogarithmic, at least asymptotically, for $c=O(1)$ ). The construction is non-uniform, assuming a certain small expander which gets used in a graph product. Siegel [46] states about his scheme that it is "far too slow for any practical application".

The independence measure has long been central to the study of randomized algorithms. For example, [29] considers variants of QuickSort, [1] consider the maximal bucket size for hashing with chaining, and $[28,19]$ consider Cuckoo hashing. In several cases [1, 19, 29], it is proved that linear transformations $x \mapsto((a x+b) \bmod p)$ do not suffice for good performance, hence that 2-independence is not in itself sufficient.

This paper surveys a family of "tabulation" based hash function that like Siegel's hash function use $O\left(u^{1 / c}\right)$ space for some constant $c$, but which are simple and practical, and offer strong probabilistic guarantees for many popular randomized algorithms despite having low independence. We start with the simplest and fastest tabulation scheme, and move later to more complicated schemes with stronger probabilistic guarantees.

We note that there has been several previous works using tabulation to construct efficient hash functions (see, e.g., $[18,20]$ ), but the schemes discussed here are simpler and more efficient. More detailed comparisons with previous works are found in the papers surveyed.

\section{Simple tabulation}

The first scheme we consider is simple tabulation hashing where the hash values are $r$-bit numbers. Our goal is to hash keys from $\mathcal{U}=[u]$ into the range $\mathcal{R}=\left[2^{r}\right]$. In tabulation hashing, a key $x \in[u]$ is interpreted as a vector of $c>1$ "characters" from the alphabet $\Sigma=\left[u^{1 / c}\right]$, i.e., $x=\left(x_{0}, \ldots, x_{c-1}\right) \in \Sigma^{c}$. As a slight abuse of notation, we shall sometimes use $\Sigma$ instead of $|\Sigma|$ to denote the size of the alphabet when the context makes this meaning clear. This matches the classic recursive set-theoretic definition of a natural as the set of smaller naturals. 
For "simple tabulation hashing" we initialize independent random character tables $h_{0}, \ldots, h_{c-1}: \Sigma \rightarrow$ $\mathcal{R}$. The hash $h(x)$ of a key $x=\left(x_{0}, \ldots, x_{c-1}\right)$ is computed as:

$$
h(x)=\bigoplus_{i \in[c]} h_{i}\left[x_{i}\right]
$$

Here $\oplus$ denotes bit-wise exclusive-or. This is a well-known scheme dating back at least to Zobrist [53]. For him a character position corresponds to a position on a game board, and the character is the piece at the position. If the piece at a position $i$ changes from $x_{i}$ to $x_{i}^{\prime}$, he updates the overall hash value $h$ to $h^{\prime}=h \oplus h_{i}\left[x_{i}\right] \oplus h_{i}\left[x_{i}^{\prime}\right]$.

It is easy to see that simple tabulation is 3-independent, for if we have a set $X$ of two or three keys, then there must be a position $i \in[c]$ where one key $x$ has a character $x_{i}$ not shared with any other key in $X$. This means that $x$ is the only key in $X$ whose hash value depends on $h_{i}\left[x_{i}\right]$, so the hash value of $x$ is independent of the other hash values from $X$. On the other hand, simple tabulation is not 4-independent. Given 4 keys $\left(a_{0}, b_{0}\right),\left(a_{1}, b_{0}\right),\left(a_{0}, b_{1}\right),\left(a_{1}, b_{1}\right)$, no matter how we fill our tables, we have

$$
h\left(a_{0}, b_{0}\right) \oplus h\left(a_{1}, b_{0}\right) \oplus h\left(a_{0}, b_{1}\right) \oplus h\left(a_{1}, b_{1}\right)=0 .
$$

Thus, given the hash values of any of three of the keys, we can uniquely determine the fourth hash value.

In our context, we assume that the number $c=O(1)$ of character positions is constant, and that character tables fit in fast cache. Justifying this assumption, recall that if we have $n$ keys from a very large universe, we can first do a universe reduction, applying a universal hashing function [9] into an intermediate universe of size $u=n^{O(1)}$, expecting no collisions (sometimes, as in [48], we can even accept some collisions from the first universal hashing, and hence use an intermediate universe size closer to $n$ ). Now, for any desired small constant $\varepsilon>0$, we get down to space $O\left(n^{\varepsilon}\right)$ picking $c=O(1)$ so that $\Sigma=u^{1 / c}<n^{\varepsilon}$. We shall refer to the lookups in the $h_{i}$ as "character lookups", emphasizing that they are expected to be much faster than a general lookup in a table of size $n$.

Putting things into a practical perspective (this paper claims practical schemes with strong theoretical guarantees), in the experiments from [41, 49], for 32-bit keys, simple tabulation with 4 character lookups took less than $5 \mathrm{~ns}$ whereas a single memory lookup in a $4 \mathrm{MB}$ table took more than 120ns. Character lookups were thus about 100 times faster than general lookups! We note that the character lookups parallelize easily and this may or may not have been exploited in the execution (we just wrote portable C-code, leaving the rest to compiler and computer). Simple tabulation was only $60 \%$ slower than the 2-independent multiply-shift scheme from [16] which for 32-bit keys is dominated by a single 64-bit multiplication. However, simple tabulation is 3-independent and in experiments from [41], it was found to be more than three times faster than 3-independent hashing implemented by a degree-2 polynomial tuned over the Mersenne prime field $\mathbb{Z}_{2^{61}-1}$. The high speed of simple tabulation conforms with experiments on much older architectures [47]. Because cache is so critical to computation, most computers are configured with a very fast cache, and this is unlikely to change.

Usually it is not a problem to fill the character tables $h_{0}, \ldots, h_{c-1}$ with random numbers, e.g., downloading them from http: / / random.org which is based on atmospheric noise. However, for the theory presented here, it would suffice to fill them with a strong enough pseudo-random number generator (PRG), like a $(\lg u)$-independent hash function, e.g., using the new fast generation from [11]. The character tables just need to point to an area in memory with random bits, and this could be shared across many applications. One could even imagine computers configured with random bits in some very fast read-only memory allowing parallel access from multiple cores. 
In [41] simple tabulation hashing was proved to have much more power than suggested by its 3independence. This included fourth moment bounds, min-wise hashing, random graph properties necessary in cuckoo hashing [39], and Chernoff bounds for distributing balls into many bins. The details follow below.

Concentration bounds First we consider using simple tabulation hashing to distribute $n$ balls into $m=2^{r}$ bins, that is, assuming that the balls have keys from $[u]$, we are using a simple tabulation hash function $h:[u] \rightarrow[m]$. In a hash table with chaining, the balls in a bin would be stored in a linked list.

Consider the number $X$ of balls landing in a given bin. We have $\mu=\mathrm{E}[X]=n / m$. Pătraşcu and Thorup [41] have proved that, w.h.p., we get a Chernoff-style concentration on $X$. First recall the classic Chernoff bounds [36, $\S 4]$ for full randomness. On the upper bound side, we have [36, Theorem 4.1]

$$
\operatorname{Pr}[X \geq(1+\delta) \mu] \leq\left(\frac{e^{\delta}}{(1+\delta)^{(1+\delta)}}\right)^{\mu}\left[\leq \exp \left(-\delta^{2} \mu / 3\right) \text { for } \delta \leq 1\right]
$$

The corresponding probabilistic lower bound [36, Proof of Theorem 4.2] for $\delta \leq 1$ is

$$
\operatorname{Pr}[X \leq(1-\delta) \mu] \leq\left(\frac{e^{-\delta}}{(1-\delta)^{(1-\delta)}}\right)^{\mu}\left[\leq \exp \left(-\delta^{2} \mu / 2\right) \text { for } \delta \leq 1\right]
$$

We note that in connection with hash tables, we are often not just interested in a given bin, but rather we care about the bin that a specific query ball lands in. This is why the hash of the query ball is involved in the theorem below with Chernoff-style bounds for simple tabulation.

Theorem 1 ([41]) Consider hashing $n$ balls into $m \geq n^{1-1 /(2 c)}$ bins by simple tabulation (recall that $c=O(1)$ is the number of characters). Define $X$ as the number of regular balls that hash into a given bin or a bin chosen as a function of the bin $h(q)$ of an additional query ball $q$. The following probability bounds hold for any constant $\gamma$ :

$$
\begin{aligned}
& \operatorname{Pr}[X \geq(1+\delta) \mu] \leq\left(\frac{e^{\delta}}{(1+\delta)^{(1+\delta)}}\right)^{\Omega(\mu)}+1 / m^{\gamma} \\
& \operatorname{Pr}[X \leq(1-\delta) \mu] \leq\left(\frac{e^{-\delta}}{(1-\delta)^{(1-\delta)}}\right)^{\Omega(\mu)}+1 / m^{\gamma}
\end{aligned}
$$

With $m \leq n$ bins (including $m<n^{1-1 /(2 c)}$ ), every bin gets

$$
n / m \pm O\left(\sqrt{n / m} \log ^{c} n\right) .
$$

balls with probability $1-n^{-\gamma}$.

Contrasting the standard Chernoff bounds, we see that Theorem 1 (5) and (6) can only provide polynomially small probability, i.e. at least $m^{-\gamma}$ for any desired constant $\gamma$. This corresponds to if we had $\Theta(\log m)$-independence in the Chernoff bound from [43]. In addition, the exponential dependence on $\mu$ is reduced by a constant which depends (exponentially) on the constants $\gamma$ and $c$.

The upper bound (5) implies that any given bin has $O(\lg n / \lg \lg n)$ balls w.h.p., but then this holds for all $m$ bins w.h.p. Simple tabulation is the simplest and fastest constant time hash function to achieve this fundamental property.

Complementing the above Chernoff-style bound, Dahlgaard et al. [13] have proved that we also get the $k^{\text {th }}$ moment bounds normally associated with $k$-independence. 
Theorem 2 ([13]) With the same setup as in Theorem 1, for any constant $k=O(1)$,

$$
\mathrm{E}\left[(X-\mu)^{k}\right]=O\left(\mu+\mu^{k / 2}\right) .
$$

For $k \leq 4$, this bound was proved in [41], and disallowing the dependence on a query ball, it was also proved in [6], again for $k \leq 4$. Compelling applications of $4^{\text {th }}$ moment bounds are given by [2], [29], and [38]. In [29], it was shown that any hash function with a good $4^{\text {th }}$ moment bound suffices for a non-recursive version of QuickSort, routing on the hypercube, etc. In [2], the $4^{\text {th }}$ moment is used to estimate the $2^{\text {nd }}$ moment of a data stream. In [38], limited $4^{\text {th }}$ moment is shown to imply constant expected performance for linear probing. The applications in $[29,38]$ both require dependence of a query bin.

Since $k^{\text {th }}$ moment bounds is one of the main ways $k$-independence is used, it is nice that they are achieved by simple tabulation which is only 3 -independent.

Linear probing Theorem 1 is used in [41] to get bounds for linear probing. Linear probing is a classic implementation of hash tables. It uses a hash function $h$ to map a set of $n$ keys into an array of size $m$. When inserting $x$, if the desired location $h(x) \in[m]$ is already occupied, the algorithm scans $h(x)+1, h(x)+$ $2, \ldots, m-1,0,1, \ldots$ until an empty location is found, and places $x$ there. The query algorithm starts at $h(x)$ and scans until it either finds $x$, or runs into an empty position, which certifies that $x$ is not in the hash table. When the query search is unsuccessful, that is, when $x$ is not stored, the query algorithm scans exactly the same locations as an insert of $x$. A general bound on the query time is hence also a bound on the insertion time.

This classic data structure is one of the most popular implementations of hash tables, due to its unmatched simplicity and efficiency. The practical use of linear probing dates back at least to 1954 to an assembly program by Samuel, Amdahl, Boehme (c.f. [31]). On modern architectures, access to memory is done in cache lines (of much more than a word), so inspecting a few consecutive values typically translates into a single memory access. Even if the scan straddles a cache line, the behavior will still be better than a second random memory access on architectures with prefetching. Empirical evaluations [5, 26, 39] confirm the practical advantage of linear probing over other known schemes, while cautioning [26, 51] that it behaves quite unreliably with weak hash functions.

Linear probing was shown to take expected constant time for any operation in 1963 by Knuth [30], in a report which is now regarded as the birth of algorithm analysis. This analysis, however, assumed a truly random hash function. However, Pagh et al. [38] showed that just 5-independence suffices for this expected constant operation time. In [40], 5-independence was proved to be best possible, presented a concrete combination of keys and a 4-independent random hash function where searching certain keys takes $\Omega(\log n)$ expected time.

In [41], the result from [38] is strengthened for more filled linear probing tables, showing that if the table size is $m=(1+\varepsilon) n$, then the expected time per operation is $O\left(1 / \varepsilon^{2}\right)$, which asymptotically matches the bound of Knuth [30] with truly random hashing. More important for this paper, [41] proved that this performance bound also holds with simple tabulation hashing.

In fact, for simple tabulation, we get quite strong concentration results for the time per operation, e.g,, constant variance for constant $\varepsilon$. For contrast, with 5-independent hashing, the variance is only known to be $O(\log n)[38,51]$.

Some experiments are done in [41] comparing simple tabulation with the fast 2-independent multiplyshift scheme from [16] in linear probing. For simple inputs such as consecutive integers, the performance was extremely unreliable with the 2-independent hashing, but with simple tabulation, everything worked perfectly as expected from the theoretical guarantees. 
Cuckoo hashing In cuckoo hashing [39] we use two tables of size $m \geq(1+\varepsilon) n$ and independent hash functions $h_{0}$ and $h_{1}$ mapping the keys to these two tables. Cuckoo hashing succeeds if we can place every key in one of its two hash locations without any collision. We can think of this as a bipartite graph with a set for each table and an edge $\left(h_{0}(x), h_{1}(x)\right)$ for each key $x$. Cuckoo hashing fails exactly if this graph has a component with more edges than vertices. With truly random hashing, this bad event happens with probability $\Theta\left(\frac{1}{n}\right)$. Pătraşcu and Thorup [41] study the random graphs induced by simple tabulation, and obtain a rather unintuitive result: the worst failure probability is inversely proportional to the cube root of the set size.

Theorem 3 ([41]) Any set of $n$ keys can be placed in two tables of size $m=(1+\varepsilon)$ by cuckoo hashing and simple tabulation with probability $1-O\left(n^{-1 / 3}\right)$. There exist sets on which the failure probability is $\Omega\left(n^{-1 / 3}\right)$.

Thus, cuckoo hashing with simple tabulation is an excellent construction for a static dictionary. The dictionary can be built (in linear time) after trying $O(1)$ independent hash functions w.h.p., and later every query runs in constant worst-case time with two probes. We note that even though cuckoo hashing requires two independent hash functions, these essentially come for the cost of one in simple tabulation: the pair of hash codes can be stored consecutively, in the same cache line, making the running time comparable with evaluating just one hash function.

In the dynamic case, Theorem 3 implies that we expect $\Omega\left(n^{4 / 3}\right)$ updates between failures requiring a complete rehash with new hash functions.

Minwise independence In [41] it is shown that simple tabulation is $\varepsilon$-minwise independent, for a vanishingly small $\varepsilon$ (inversely polynomial in the set size $n$ ). This takes $\Theta(\log n)$-independence by general techniques [27, 40]. More precisely, we have

Theorem 4 ([41]) Consider a set $S \subseteq \Sigma^{c}$ of $n=|S|$ keys and $q \in S$. If $h: \Sigma^{c} \rightarrow[m], m \geq n^{1+1 / c}$ is implemented by simple tabulation:

$$
\operatorname{Pr}[h(q)=\min h(S)]=\frac{1 \pm \varepsilon}{n}, \quad \text { where } \varepsilon=O\left(\frac{\lg ^{2} n}{n^{1 / c}}\right) .
$$

The classic application of $\varepsilon$-minwise hashing of Broder [8,7] is the estimation of Jaccard set similarity $|A \cap B| /|A \cup B|$. Ignoring the probability of collisions in the minimum hash value, we get

$$
\begin{aligned}
\operatorname{Pr}[\min h(A)= & \min h(B)] \\
& =\sum_{x \in A \cap B} \operatorname{Pr}[h(x)=\min h(A \cup B)] \\
& =\frac{|A \cap B|}{|A \cup B|} \cdot\left(1 \pm \widetilde{O}\left(\frac{1}{|A \cup B|^{1 / c}}\right)\right) .
\end{aligned}
$$

For better bounds on the probabilities, we would make multiple experiments with independent hash functions, yet this cannot eliminate the bias $\varepsilon$. 
The power of two choices The power of two choices is a standard scheme for placing balls into bins where each ball hashes to two bins, and is placed in the lightest loaded one (see [33] for a survey). When placing $n$ balls into $n$ bins, using the two-choice paradigm with truly random hash functions, the maximum load of any bin is $\lg \lg n+O(1)$ w.h.p. [4]. Dahlgaard et al. [14] have proved that simple tabulation gives a maximum $\operatorname{load}$ which is $\lg \lg n+O(1)$ in expectation and $O(\log \log n)$ w.h.p.. This is the simplest constant time hashing scheme known to offer such strong two-choice load balancing.

Weakness with small numbers As described above, simple tabulation has much more power than suggested by its 3-independence. However, there are also some weaknesses. For example, in the Chernoff-style bounds (5) and (6) from Theorem 1, we have an additive error probability of $1 / m^{\gamma}$ when hashing into $m$ bins. Here $\gamma$ is an arbitrarily large constant, so this is fine when $m$ is large. However, this is not good if $m$ is small, e.g., $m=2$ as when we toss a coin. A somewhat related problem is that our bias $\varepsilon$ in $\varepsilon$-minwise independence in (8) is $\tilde{O}\left(1 / n^{1 / c}\right)$ where $n$ is the size of the set considered. This is fine if the set is large, but not if the set is small. Both of these problems and more will be addressed by twisted tabulation described below.

\section{Twisted tabulation}

We will now consider twisted tabulation proposed by Pătraşcu and Thorup in [42]. It adds a quick twist to simple tabulation, leading to more general distributional properties, including Chernoff bounds that also work for few bins and better minwise hashing that also works well for small sets. For $i=1, \ldots, c-1$, we expand the entries of $h_{i}$ with a random character called the "twister". More precisely, for $i>0$, we now have random tables $h_{i}^{\star}: \Sigma \rightarrow \Sigma \times \mathcal{R}$. This adds $\lg \Sigma$ bits to each entry of the table (in practice, we want entries to have bit lengths like 32 or 64 , so depending on $\mathcal{R}$, the twister may, or may not, increase the actual entry size). The table $h_{0}: \Sigma \rightarrow \mathcal{R}$ is kept unchanged. The hash function is now computed in two statements:

$$
\begin{aligned}
\left(t, h_{>0}\right) & =\bigoplus_{i=1}^{c-1} h_{i}^{\star}\left[x_{i}\right] ; \\
h(x) & =h_{>0} \oplus h_{0}\left[x_{0} \oplus t\right] .
\end{aligned}
$$

Figure 1 contains the C-code for simple and twisted tabulation.

The highlight of twisted tabulation is its minimalistic nature, adding very little to the cost of simple tabulation (but, as we shall see, with significantly stronger guarantees). Twisted tabulation uses exactly $c$ character lookups into tables with $\Sigma$ entries, just like simple tabulation, though with larger entries. Essentially twisted tabulation only differs from simple tabulation by two $\mathrm{AC}^{0}$ operations, so we would expect it to be almost as fast as simple tabulation (whose practicality has long been established). This was confirmed experimentally in [42], where twisted tabulation was less than $30 \%$ slower than simple tabulation, and still nearly three times faster than a second degree polynomial.

When we discuss properties of twisted tabulation, we view keys $x=\left(x_{0}, \ldots, x_{c-1}\right)$ as composed of a head, $\operatorname{head}(x)=x_{0}$ and a tail, tail $(x)=\left(x_{1}, \ldots, x_{c-1}\right)$. We refer to the following implementation of twisted tabulation which is less efficient but mathematically equivalent to (9):

1. Pick a simple tabulation hash function $h^{\tau}: \Sigma^{c-1} \rightarrow \Sigma$ from $c-1$ characters to 1 character. This corresponds to the twister components of $h_{1}^{\star}, \ldots, h_{c-1}^{\star}$. Applying $h^{\tau}$ to the tail of a key $x$, we get 


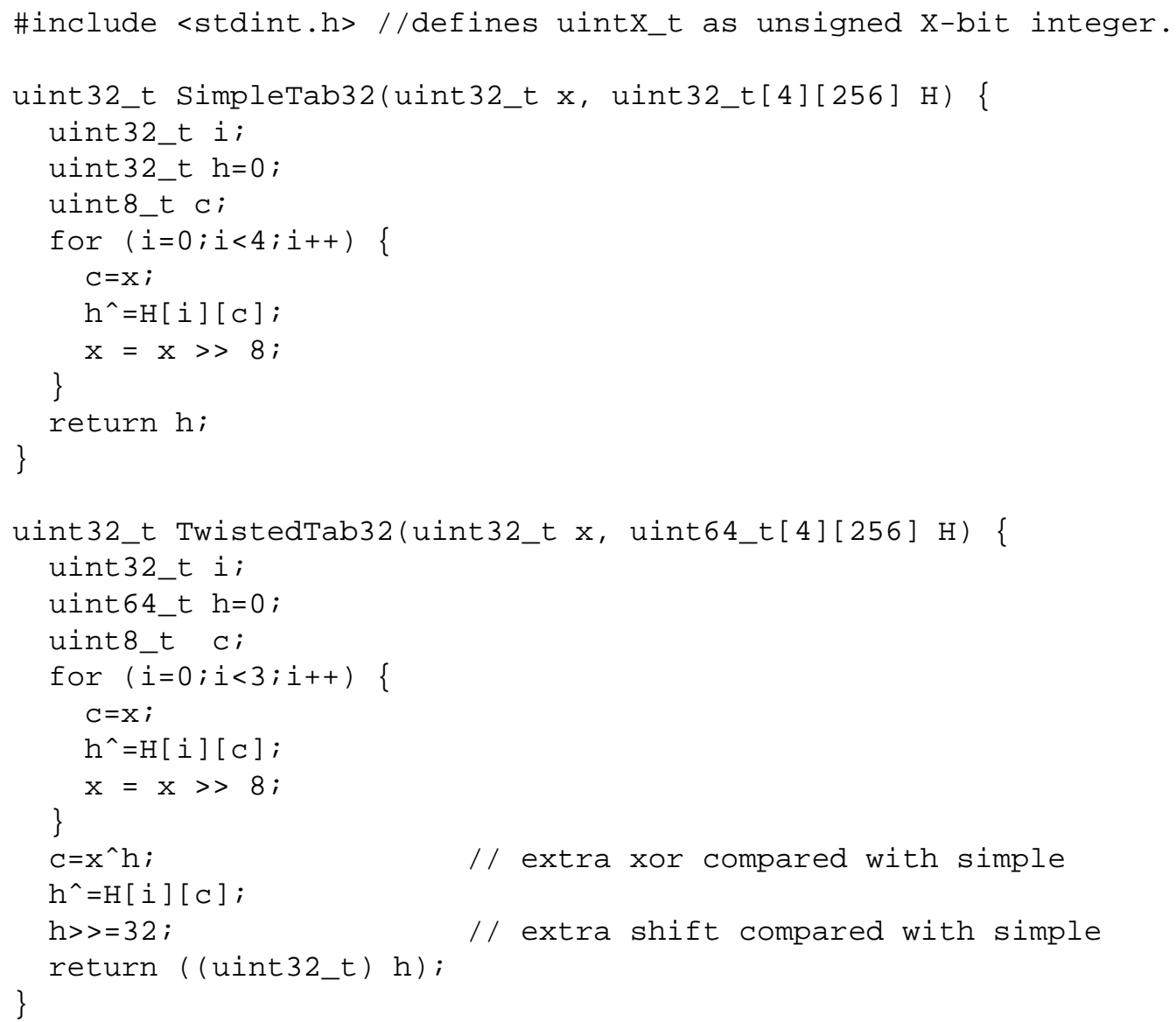

Figure 1: C-code for simple and twisted tabulation for 32-bit keys assuming a pointer $\mathrm{H}$ to some randomly filled storage (4KB for simple and $8 \mathrm{~KB}$ for twisted). 
the combined twister $t=h^{\tau}(\operatorname{tail}(x))$, the "twisted head" $x_{0} \oplus t$, and the "twisted key" $h^{\mathcal{T}}(x)=$ $\left(x_{0} \oplus t, x_{1}, \ldots, x_{c-1}\right)$.

2. Pick a simple tabulation hash function $h^{\mathcal{S}}: \Sigma^{c} \rightarrow \mathcal{R}$ (where $\mathcal{R}$ was the desired output range). This corresponds to $h_{0}$ and for $i>0$, the non-twister component $h_{i}$ of $h_{i}^{\star}$ (remember that tails are not touched by twisting). The twisted tabulation hash function is then $x \mapsto h^{\mathcal{S}}\left(h^{\mathcal{T}}(x)\right)$.

For all the results presented here, it does not matter which character we view as the head. Above it is the first character, but sometimes it is more efficient if it is the last least significant character.

As noted in [42], the twisting by $h^{\mathcal{T}}$ can be seen as a single-round Feistel permutation where the hash function is simple tabulation. Because twisting is a permutation, twisted tabulation inherit from simple tabulation any (probabilistic) property that holds regardless of concrete key values, e.g., the above Cuckoo hashing and the power of two choices. Like simple tabulation, twisted tabulation is only 3-independent, but it does have some more general distributional guarantees, which we explain in detail below.

Chernoff Bounds Chernoff bounds play a prominent role in the design of randomized algorithms [36, $\S 4]$. The Chernoff-style bounds from Theorem 1 where limited in that they only really worked for throwing balls into a large number $m$ of bins. In [42], Pătraşcu and Thorup prove the following far more general Chernoff-style bounds for twisted tabulation.

Theorem 5 ([42]) Choose a random c-character twisted tabulation hash function $h=h^{\mathcal{S}} \circ h^{\mathcal{T}}:[u] \rightarrow[u]$, $[u]=\Sigma^{c}$. For each key $x \in[u]$ in the universe, we have an arbitrary "value function" $v_{x}:[u] \rightarrow[0,1]$ assigning a value $V_{x}=v_{x}(h(x)) \in[0,1]$ to $x$ for each hash value. Define $V=\sum_{x \in[u]} V_{x}$ and $\mu=$ $\sum_{x \in[u]} \mu_{x}$ where $\mu_{x}=\mathrm{E}\left[v_{x}(h(x))\right]$. Let $\gamma$, c and $\varepsilon>0$ be constants. Then for any $\mu<\Sigma^{1-\varepsilon}$ and $\delta>0$, we have:

$$
\begin{aligned}
& \operatorname{Pr}[V \geq(1+\delta) \mu] \leq\left(\frac{e^{\delta}}{(1+\delta)^{(1+\delta)}}\right)^{\Omega(\mu)}+1 / u^{\gamma} \\
& \operatorname{Pr}[V \leq(1-\delta) \mu] \leq\left(\frac{e^{-\delta}}{(1-\delta)^{(1-\delta)}}\right)^{\Omega(\mu)}+1 / u^{\gamma} .
\end{aligned}
$$

Moreover, for any $\mu \geq \sqrt{\Sigma}$ (including $\mu \geq \Sigma^{1-\varepsilon}$ ), with probability $1-u^{-\gamma}$,

$$
V=\mu \pm \widetilde{O}(\sqrt{\mu}) .
$$

If we have a given distinguished query key $q \in[u]$, the above bounds hold even if we condition everything on $h(q)=$ a for any given $a \in[u]^{1}$.

The statement of Theorem 5 may seem a bit cryptic, so before proceeding, we will show that it improves the simple tabulation bounds from Theorem 1. Those bounds considered a set $S$ of $n$ balls or keys mapped into $m$ bins, and had an error bound of $m^{-\gamma}$. The error is here improved to $u^{-\gamma}$.

We now do the translation. Discounting all irrelevant keys $x \in[u] \backslash S$, we zero their value functions, setting $v_{x}(\cdot)=0$. Also, we define the bin hash function from Theorem 1 as $h^{\prime}(x)=h(x) \bmod m$, noting that $m \uparrow u$ since both are powers of two. Theorem 1 studies the number of balls landing a bin $b$ which may be a function of the bin $h^{\prime}(q)$ of a query ball $q \in[u] \backslash S$. Thanks to the last statement of Theorem

\footnotetext{
${ }^{1}$ This last statement conditioning on $h(q)=a$ was not proved in [42], but it is an easy extension provided in Appendix A.
} 
5, we can condition on any value $a$ of $h(q)$, which determines $h^{\prime}(q)$ and hence $b$. Now, for $x \in S$, define $V_{x}=v_{x}(h(x))=1$ if $h^{\prime}(x)=b$; 0 otherwise. Now $V=\sum_{x \in[u]} V_{x}$ is the variable $X$ from Theorem 1, and (5) and (6) follow from (10) and (11), but with the improved error $u^{-\gamma}$. This is important when $m$ is small, e.g., if $m=2$ corresponding to unbiased coin tosses.

A different illustration of the versatility of Theorem 5 is if we want each key $x \in[u]$ to be sampled with some individual sampling probability $p_{x}$. In this case we have no distinguished query key, and we can just define $v_{x}(y)=1$ if $y<p_{x} \cdot m$; otherwise $v_{x}(y)=0$. Since $h(x)$ is uniform in $[m]$, we have that $x$ is sampled with $V_{x}=v_{x}(h(x))=1$ with probability $\tilde{p}_{x}=\left\lceil p_{x} m\right\rceil / m$. The number $V=\sum_{x \in[u]} V_{x}$ of samples is now concentrated according to (10) and (11).

Minwise independence Concerning minwise hashing, Dahlgaard and Thorup [15] have proved that twisted tabulation yields the following strengthening of Theorem 4 for simple tabulation.

Theorem 6 ([15]) Consider a set $S \subseteq \Sigma^{c}$ of $n=|S|$ keys and $q \in S$. If $h: \Sigma^{c} \rightarrow[m], m \geq n u^{1 / c}$ is implemented by twisted tabulation then:

$$
\operatorname{Pr}[h(q)=\min h(S)]=\frac{1 \pm \varepsilon}{n}, \quad \text { where } \varepsilon=O\left(\frac{\lg ^{2} u}{u^{1 / c}}\right)
$$

The important difference is that the bias $O\left(\frac{\lg ^{2} n}{n^{1 / c}}\right)$ from Theorem 4 is replaced by $O\left(\frac{\lg ^{2} u}{u^{1 / c}}\right)$ which is small regardless of the set size. Such an absolutely small bias generally requires $\Omega(\log u)$-independence [40].

Short range amortization for chaining and linear probing We now switch to a quite different illustration of the power of twisted tabulation hashing from [42]. Consider linear probing in a half-full hash table with $n$ keys. Out of $\sqrt{n}$ operations, we expect some to take $\Omega(\log n)$ time. Nevertheless we show that any window of $\log n$ operations on distinct keys is executed in $O(\lg n)$ time with high probability. This also holds for the simpler case of chaining.

The general point is that for any set of stored keys and any set of window keys, the operation times within the window are sufficiently independent that the average concentrates nicely around the expected constant operation time. Such concentration of the average should not be taken for granted with real hash functions. In [40] are input examples for linear probing with multiplication-shift hashing [16] such that if one operation is slow, then most operations are slow. In [42] is presented a parallel universe construction causing similar problems for simple tabulation. As stated above, twisted tabulation does, however, provide sufficient independence, and we expect this to prove useful in other applications.

Pseudo-random numbers generators Like any hash function, twisted tabulation naturally implies a pseudo-random numbers generators (PRG) with the pseudo-random sequence $h(0), h(1), \ldots$. For maximal efficiency, we use the last and least significant character as the head. Thus, a key $x=\left(x_{c-1}, \ldots, x_{0}\right) \in \Sigma^{c}$ has head $(x)=x_{0}$ and tail $(x)=x_{>0}=\left(x_{c-1}, \ldots, x_{1}\right)$. For twisted tabulation, we use a simple tabulation function $h^{\star}: \Sigma^{c-1} \rightarrow \Sigma \times\left[2^{r}\right]$ and a character function $h_{0}: \Sigma \rightarrow\left[2^{r}\right]$, and then $h(x)$ is computed, setting $\left(t, h_{>0}\right)=h^{\star}\left(x_{>0}\right)$ and $h(x)=h_{0}\left[x_{0} \oplus t\right] \oplus h_{>0}$. We now think of $x$ as the pair $\left(x_{>0}, x_{0}\right) \in \Sigma^{c-1} \times \Sigma$. As we increase the index $x=0,1, \ldots, \Sigma-1, \Sigma, \Sigma+1, \ldots=(0,0),(0,1), \ldots,(0, \Sigma-1),(1,0),(1,1), \ldots$, the tail $x_{>0}$ only increases when $x_{0}$ wraps around to 0 - once in every $\Sigma$ calls. We therefore store $\left(t, h_{>0}\right)=$ $h^{\star}\left(x_{>0}\right)$ in a register, recomputing it only when $x_{>0}$ increases. Otherwise we compute $h(x)=h_{0}\left[x_{0} \oplus t\right] \oplus$ $h_{>0}$ using just one character lookup and two $\oplus$-operations. We found this to be exceedingly fast: as fast as 
a single multiplication and 4 times faster than the standard random number generator random () from the GNU C library which has almost no probabilistic guarantees. Besides being faster, the twisted PRG offers the powerful distributional guarantees discussed above.

As an alternative implementation, we note that $h^{\star}$ is itself applied to consecutive numbers $x_{>0}=$ $0,1,2, \ldots$, so $h^{\star}$ can also be implemented as a PRG. The $h^{\star}$-PRG is only applied once for every $\Sigma$ numbers generated by $h$, so the $h^{\star}$-PRG can be much slower without affecting the overall performance. Instead of implementing $h^{\star}$ by simple tabulation, we could implement it with any logarithmically independent PRG, thus not storing any tables for $h^{\star}$, but instead generating each new value $h^{\star}\left(x_{>0}\right)$ on the fly as $x_{>0}$ increases. We can view this as a general conversion of a comparatively slow but powerful PRG into an extremely fast one preserving the many probabilistic properties of twisted tabulation. The recent PRG of Christiani and Pagh [11] would be a good choice for the $h^{\star}$-PRG if we do not want to implement it with simple tabulation.

Randomized Algorithms and Data Structures When using the twisted PRG in randomized algorithms [36], we get the obvious advantage of the Chernoff-style bounds from Theorem 5 which is one of the basic techniques needed [36, $\S 4]$. The $\varepsilon$-minwise hashing from Theorem 6 with $\varepsilon=\widetilde{O}\left(1 / u^{1 / c}\right)$ is important in contexts where we want to assign randomized priorities to items. A direct example is treaps [44]. The classic analysis [36, $\S 8.2]$ of the expected operation time is based on each key in an interval having the same chance of getting the lowest priority. Assigning the priorities with an $\varepsilon$-minwise hash function, expected cost is only increased by a factor $(1+\varepsilon)$ compared with the unrealistic case of true randomness. In static settings, we can also use this to generate a random permutation, sorting items according to priorities. This sorting itself takes linear time since we essentially only need to look at the $\log n$ most significant bits to sort $n$ priorities. Using this order to pick items, we get that classic algorithms like QuickSort [36, §1] and Binary Planar Partitions $[36, \S 1.3]$ perform within an expected factor $(1+\varepsilon)$ of what they would with true randomness. With $\varepsilon=\widetilde{O}\left(1 / u^{1 / c}\right)$ as with our twisted PRG, this is very close the expected performance with true randomness.

\section{Double tabulation and high independence}

Thorup [50] has shown that simple tabulation can also be used to get high independence if we apply it twice. More precisely, we consider having two independent simple tabulation functions $h_{0}: \Sigma^{c} \rightarrow \Sigma^{d}$ and $h_{1}: \Sigma^{d} \rightarrow\left[2^{r}\right]$, and then the claim is that $h_{1} \circ h_{0}$ is likely to be highly independent. The main point from [50] is that the first simple tabulation $h_{0}$ is likely to have an expander-type property.

More precisely, given a function $f:[u] \rightarrow \Sigma^{d}$, a key set $X \subseteq[u]$ has a unique output character if there is a key $x \in X$ and a $j \in[d]$ and such that for all $y \in X \backslash\{x\}, h(y)_{j} \neq h(x)_{j}$, that is, the $j$ th output character is unique to some $x$ in $X$. We say that $f$ is $k$-unique if each non-empty key set $Y \subseteq[u]$ of size at most $k$ has a unique output character. Siegel [46] noted that if $f$ is $k$-unique and $h_{1}: \Sigma^{d} \rightarrow\left[2^{r}\right]$ is a random simple tabulation function, then $h_{1} \circ f:[u] \rightarrow\left[2^{r}\right]$ is $k$-independent. The main technical result from [50] is

Theorem 7 ([50]) Consider a random simple tabulation function $h_{0}: \Sigma^{c} \rightarrow \Sigma^{d}$. Assume $c=\Sigma^{o(1)}$ and $(c+d)^{c}=\Sigma^{o(1)}$. Let $k=\Sigma^{1 /(5 c)}$. With probability $1-o\left(\Sigma^{2-d /(2 c)}\right)$, the function $h_{0}$ is $k$-unique. More concretely for 32-bit keys with 16-bit characters, $h_{0}$ is 100-unique with probability $1-1.5 \times 10^{-42}$.

Assuming that $h_{0}$ is $k$-unique, if $h_{1}: \Sigma^{d} \rightarrow\left[2^{r}\right]$ is a random simple tabulation function, then $h_{1} \circ h_{0}$ is $k$-independent.

This construction for highly independent hashing is much simpler than that of Siegel [46] mentioned in Section 1, and for $d=O(c)$, the evaluation takes $O(c)$ time as opposed to the $O(c)^{c}$ time used by Siegel. 
Complementing the above result, Dahlgaard et al. [13] have proved that double tabulation is likely to be truly random for any specific set $S$ with less than $(1-\Omega(1)) \Sigma$ keys:

Theorem 8 ([13]) Given a set $S \subseteq[u]$ of size $(1-\Omega(1)) \Sigma$, consider two random simple tabulation function $h_{0}: \Sigma^{c} \rightarrow \Sigma^{d}$ and $h_{1}: \Sigma^{d} \rightarrow\left[2^{r}\right]$. With probability $1-O\left(\Sigma^{1-\lfloor d / 2\rfloor}\right)$, every non-empty subset $X \subseteq S$ gets a unique output character with $h_{0}$, and then the double tabulation function $h_{1} \circ h_{0}$ is fully random over $S$.

It is interesting to compare Theorem 8 and Theorem 7. Theorem 8 holds for one large set while Theorem 7 works for all small sets. Also, Theorem 8 with $d=4$ "derived" characters gets essentially the same error probability as Theorem 7 with $d=6 c$ derived characters.

Siegel [46] has proved that with space $\Sigma$, we cannot in constant time hope to get independence higher than $\Sigma^{1-\Omega(1)}$, which is much less than the size of the given set in Theorem 8 .

Theorem 8 provides an extremely simple $O(n)$ space implementation of a constant time hash function that is likely uniform on any given set $S$. This should be compared with the previous linear space uniform hashing of Pagh and Pagh [37, §3]. The most efficient implementation of [37, §3] uses the highly independent double tabulation from Theorem 7 a subroutine. However, as discussed earlier, double tabulation uses much more derived characters for high independence than for uniformity on a given set, so for linear space uniform hashing on a given set, it is much faster and simpler to use the double tabulation of Theorem 8 directly. We note that $[37, \S 4]$ presents a general trick to reduce the space from linear, that is, $O(n(\lg n+\lg |\mathcal{R}|))$ bits, downto $(1+\varepsilon) n \lg |\mathcal{R}|+O(n)$ bits, preserving the constant evaluation time. This reduction can also be applied to Theorem 8 so that we also get a simpler overall construction for a succinct dictionary using $(1+\varepsilon) n \lg |\mathcal{R}|+O(n)$ bits of space and constant evaluation time.

Very recently, Christiani et al. [12] have shown that we using a more elaborate recursive tabulation scheme can get quite to Siegel's lower-bound.

Theorem 9 ([12, Corollary 3]) For word-size $w$, and parameters $k$ and $c=O(w /(\log k))$, with probability $1-u^{1 / c}$, we can construct a $k$-independent hash function $h:\left[2^{w}\right] \rightarrow\left[2^{w}\right]$ in $O\left(c k u^{1 / c}\right)$ time and space that is evaluated in $O(c \log c)$ time.

In Theorem 8, we used the same space to get independence $\Sigma^{1 /(5 c)}$ and evaluation time $O(c)$, and the construction of Theorem 8 is simpler. We should thus use Theorem 9 if we need its very high independence, but if, say, logarithmic independence suffices, then Theorem 8 is the better choice.

A major open problem is get the space and independence of Theorem 9 but with $O(c)$ evaluation time, matching the lower bound of [46]. In its full generality, the lower bound from [46] says that we for independence $k$ with $c<k$ cell probes need space $\Omega\left(k(u / k)^{1 / c} c\right)$.

Invertible Bloom filters with simple tabulation Theorem 8 states that if a random simple tabulation function $h_{0}: \Sigma^{c} \rightarrow \Sigma^{d}$ is applied to a given set $S$ of size $(1-\Omega(1)) \Sigma$, then with probability $1-O\left(\Sigma^{1-\lfloor d / 2\rfloor}\right)$, every non-empty subset $X \subseteq S$ gets a unique output character. This is not only relevant for fully-random hashing. This property is also sufficient for the hash function in Goodrich and Mitzenmacher's Invertible Bloom Filters [25], which have found numerous applications in streaming and data bases [21, 22, 35]. So far Invertible Bloom Filters have been implemented with fully random hashing, but Theorem 8 shows that simple tabulation suffices for the underlying hash function.

\section{1 $k$-partitions via mixed tabulation}

The general goal of Dahlgaard et al. [13] is a hash function for $k$-partitioning a set into bins so that we get good concentration bounds when combining statistics from each bin. 
To understand this point, suppose we have a fully random hash function applied to a set $X$ of red and blue balls. We want to estimate the fraction $f$ of red balls. The idea of Minwise hashing is to sample the ball with the smallest hash value. This sample is uniformly random and is red with probability $f$. If we repeat the experiment $k$ times with $k$ independent hash functions, we get a multiset $S$ of $k$ samples with replacement from $X$ and the fraction red balls in $S$ concentrates around $f$ as we increase the number of samples.

Consider the alternative experiment using a single hash function, where we use some bits of the hash value to partition $X$ into $k$ bins, and then use the remaining bits as a local hash value. We pick the ball with the smallest hash value in each bin. This is a sample $S$ from $X$ without replacement, and again, the fraction of red balls is concentrated around $f$.

The big difference between the two schemes is that the second one runs $\Omega(k)$ times faster. In the first experiment, each ball participated in $k$ independent experiments, but in the second one with $k$-partitions, each ball picks its bin, and then only participates in the local experiment for that bin. Thus with the $k$ partition, essentially, we get $k$ experiments for the price of one.

This generic idea has been used for different types of statistics. Flajolet and Martin [23] introduced it to count the number of distinct items in a multiset, Charikar et al. [10] used it in their count sketches for fast estimation of the second moment of a data stream, and recently, Li et al. [32, 45] used it for Minwise estimation of the Jaccard Similarity of two sets.

The issue is that no realistic hashing scheme was known to make a good enough $k$-partition for the above kind of statistics to make sense. The point is that the contents of different bins may be too correlated, and then we get no better concentration with a larger $k$. In the independence paradigm of Carter and Wegman [52], it would seem that we need independence at least $k$ to get sufficiently independent statistics from the different bins.

An efficient solution is based on a variant of double tabulation described below.

Mixed tabulation For Theorem 8 we may use $d=4$ even if $c$ is larger, but then $h_{0}$ will introduce many collisions. To avoid this problem we mix the schemes in mixed tabulation. Mathematically, we use two simple tabulation hash functions $h_{1}:[u] \rightarrow \Sigma^{d}$ and $h_{2}: \Sigma^{c+d} \rightarrow\left[2^{r}\right]$, and define the hash function $h(x) \mapsto h_{2}\left(x \circ h_{1}(x)\right)$, where $\circ$ denotes concatenation of characters. We call $x \circ h_{1}(x)$ the derived key, consisting of $c$ original characters and $d$ derived characters. Since the derived keys includes the original keys, there are no duplicate keys.

We note that mixed tabulation only requires $c+d$ lookups if we instead store simple tabulation functions $h_{1,2}: \Sigma^{c} \rightarrow \Sigma^{d} \times[r]$ and $h_{2}^{\prime}: \Sigma^{d} \rightarrow[r]$, computing $h(x)$ by $\left(v_{1}, v_{2}\right)=h_{1,2}(x) ; h(x)=v_{1} \oplus h_{2}\left(v_{2}\right)$. This efficient implementation is similar to that of twisted tabulation, and is equivalent to the previous definition. As long as we have at least one derived character, mixed tabulation has all the distribution properties of twisted tabulation, particularly, the Chernoff-style concentration bound from Theorem 5. At the same time, we get the full randomness from Theorem 8 for any given set $S$ of size $(1-\Omega(1)) \Sigma$. Based on these properties and more, it is proved in [13] that mixed tabulation, w.h.p., gets essentially the same concentration bounds as full randomness for all of the above mentioned statistics based on $k$-partitions.

\section{References}

[1] N. Alon, M. Dietzfelbinger, P. B. Miltersen, E. Petrank, and G. Tardos. Linear hash functions. J. ACM, 46(5):667-683, 1999. 
[2] N. Alon, Y. Matias, and M. Szegedy. The space complexity of approximating the frequency moments. Journal of Computer and System Sciences, 58(1):209-223, 1999. Announced at STOC'96.

[3] N. Alon and A. Nussboim. k-wise independent random graphs. In Proc. 49th IEEE Symposium on Foundations of Computer Science (FOCS), pages 813-822, 2008.

[4] Y. Azar, A. Z. Broder, A. R. Karlin, and E. Upfal. Balanced allocations. SIAM Journal on Computing, 29(1):180-200, 1999. Announced at STOC'94.

[5] J. R. Black, C. U. Martel, and H. Qi. Graph and hashing algorithms for modern architectures: Design and performance. In Proc. 2nd International Workshop on Algorithm Engineering (WAE), pages 3748, 1998.

[6] V. Braverman, K.-M. Chung, Z. Liu, M. Mitzenmacher, and R. Ostrovsky. AMS without 4-wise independence on product domains. In Proc. 27th Symposium on Theoretical Aspects of Computer Science (STACS), pages 119-130, 2010.

[7] A. Z. Broder, M. Charikar, A. M. Frieze, and M. Mitzenmacher. Min-wise independent permutations. Journal of Computer and System Sciences, 60(3):630-659, 2000. Announced at STOC'98.

[8] A. Z. Broder, S. C. Glassman, M. S. Manasse, and G. Zweig. Syntactic clustering of the web. Computer Networks, 29:1157-1166, 1997.

[9] L. Carter and M. N. Wegman. Universal classes of hash functions. Journal of Computer and System Sciences, 18(2):143-154, 1979. Announced at STOC'77.

[10] M. Charikar, K. Chen, and M. Farach-Colton. Finding frequent items in data streams. In Proc. 29th International Colloquium on Automata, Languages and Programming (ICALP), pages 693-703. Springer-Verlag, 2002.

[11] T. Christiani and R. Pagh. Generating k-independent variables in constant time. In Proceedings of the 55th IEEE Symposium on Foundations of Computer Science (FOCS), pages 196-205, 2014.

[12] T. Christiani, R. Pagh, and M. Thorup. From independence to expansion and back again. In Proceedings of the 47th ACM Symposium on Theory of Computing (STOC), pages 813-820, 2015.

[13] S. Dahlgaard, M. B. T. Knudsen, E. Rotenberg, and M. Thorup. Hashing for statistics over k-partitions. In Proceedings of the 56th IEEE Symposium on Foundations of Computer Science (FOCS), pages 1292-1310, 2015.

[14] S. Dahlgaard, M. B. T. Knudsen, E. Rotenberg, and M. Thorup. The power of two choices with simple tabulation. In Proceedings of the 27th ACM-SIAM Symposium on Discrete Algorithms (SODA), pages 1631-1642, 2016.

[15] S. Dahlgaard and M. Thorup. Approximately minwise independence with twisted tabulation. In Proc. 14th Scandinavian Workshop on Algorithm Theory (SWAT), pages 134-145, 2014.

[16] M. Dietzfelbinger. Universal hashing and $k$-wise independent random variables via integer arithmetic without primes. In Proc. 13th Symposium on Theoretical Aspects of Computer Science (STACS), pages 569-580, 1996. 
[17] M. Dietzfelbinger, T. Hagerup, J. Katajainen, and M. Penttonen. A reliable randomized algorithm for the closest-pair problem. Journal of Algorithms, 25(1):19-51, 1997.

[18] M. Dietzfelbinger and F. Meyer auf der Heide. A new universal class of hash functions and dynamic hashing in real time. In Proc. 17th International Colloquium on Automata, Languages and Programming (ICALP), pages 6-19, 1990.

[19] M. Dietzfelbinger and U. Schellbach. On risks of using cuckoo hashing with simple universal hash classes. In Proc. 20th ACM/SIAM Symposium on Discrete Algorithms (SODA), pages 795-804, 2009.

[20] M. Dietzfelbinger and P. Woelfel. Almost random graphs with simple hash functions. In Proc. 25th ACM Symposium on Theory of Computing (STOC), pages 629-638, 2003.

[21] D. Eppstein and M. T. Goodrich. Straggler identification in round-trip data streams via newton's identities and invertible bloom filters. Knowledge and Data Engineering, IEEE Transactions on, 23(2):297306, 2011.

[22] D. Eppstein, M. T. Goodrich, F. Uyeda, and G. Varghese. What's the difference?: efficient set reconciliation without prior context. ACM SIGCOMM Computer Communication Review, 41(4):218-229, 2011.

[23] P. Flajolet and G. N. Martin. Probabilistic counting algorithms for data base applications. Journal of Computer and System Sciences, 31(2):182-209, 1985.

[24] M. L. Fredman, J. Komlós, and E. Szemerédi. Storing a sparse table with 0(1) worst case access time. Journal of the ACM, 31(3):538-544, 1984. Announced at FOCS'82.

[25] M. T. Goodrich and M. Mitzenmacher. Invertible bloom lookup tables. In Proceedings of the 49th Allerton Conference on Communication, Control, and Computing, pages 792-799, 2011.

[26] G. L. Heileman and W. Luo. How caching affects hashing. In Proc. 7th Workshop on Algorithm Engineering and Experiments (ALENEX), pages 141-154, 2005.

[27] P. Indyk. A small approximately min-wise independent family of hash functions. Journal of Algorithms, 38(1):84-90, 2001. Announced at SODA'99.

[28] D. M. K. Jeffery S. Cohen. Bounds on the independence required for cuckoo hashing, 2009. Manuscript.

[29] H. J. Karloff and P. Raghavan. Randomized algorithms and pseudorandom numbers. Journal of the $A C M, 40(3): 454-476,1993$.

[30] D. E. Knuth. Notes on open addressing. Unpublished memorandum. See http://citeseer.ist.psu.edu/knuth63notes.html, 1963.

[31] D. E. Knuth. The Art of Computer Programming, Volume III: Sorting and Searching. Addison-Wesley, 1973.

[32] P. Li, A. B. Owen, and C.-H. Zhang. One permutation hashing. In Proc. 26th Conference on Neural Information Processing Systems (NIPS), pages 3122-3130, 2012. 
[33] M. Mitzenmacher, A. W. Richa, and R. Sitaraman. The power of two random choices: A survey of techniques and results. In P. Pardalos, S. Rajasekaran, and J. Rolim, editors, Handbook of Randomized Computing: volume 1, pages 255-312. Springer, 2001.

[34] M. Mitzenmacher and S. P. Vadhan. Why simple hash functions work: exploiting the entropy in a data stream. In Proc. 19th ACM/SIAM Symposium on Discrete Algorithms (SODA), pages 746-755, 2008.

[35] M. Mitzenmacher and G. Varghese. Biff (bloom filter) codes: Fast error correction for large data sets. In Information Theory Proceedings (ISIT), 2012 IEEE International Symposium on, pages 483-487. IEEE, 2012.

[36] R. Motwani and P. Raghavan. Randomized Algorithms. Cambridge University Press, 1995.

[37] A. Pagh and R. Pagh. Uniform hashing in constant time and optimal space. SIAM Journal on Computing, 38(1):85-96, 2008.

[38] A. Pagh, R. Pagh, and M. Ružić. Linear probing with constant independence. SIAM Journal on Computing, 39(3):1107-1120, 2009. Announced at STOC'07.

[39] R. Pagh and F. F. Rodler. Cuckoo hashing. Journal of Algorithms, 51(2):122-144, 2004. Announced at ESA'01.

[40] M. Pătraşcu and M. Thorup. On the $k$-independence required by linear probing and minwise independence. In Proc. 37th International Colloquium on Automata, Languages and Programming (ICALP), pages 715-726, 2010.

[41] M. Pătraşcu and M. Thorup. The power of simple tabulation-based hashing. Journal of the ACM, 59(3):Article 14, 2012. Announced at STOC'11.

[42] M. Pătraşcu and M. Thorup. Twisted tabulation hashing. In Proc. 24th ACM/SIAM Symposium on Discrete Algorithms (SODA), pages 209-228, 2013.

[43] J. P. Schmidt, A. Siegel, and A. Srinivasan. Chernoff-Hoeffding bounds for applications with limited independence. SIAM Journal on Discrete Mathematics, 8(2):223-250, 1995. Announced at SODA'93.

[44] R. Seidel and C. R. Aragon. Randomized search trees. Algorithmica, 16(4/5):464-497, 1996. Announced at FOCS'89.

[45] A. Shrivastava and P. Li. Densifying one permutation hashing via rotation for fast near ne ighbor search. In Proc. 31th International Conference on Machine Learning (ICML), pages 557-565, 2014.

[46] A. Siegel. On universal classes of extremely random constant-time hash functions. SIAM Journal on Computing, 33(3):505-543, 2004. Announced at FOCS'89.

[47] M. Thorup. Even strongly universal hashing is pretty fast. In Proc. 11th ACM/SIAM Symposium on Discrete Algorithms (SODA), pages 496-497, 2000.

[48] M. Thorup. String hashing for linear probing. In Proc. 20th ACM/SIAM Symposium on Discrete Algorithms (SODA), pages 655-664, 2009.

[49] M. Thorup. Timeouts with time-reversed linear probing. In Proc. IEEE INFOCOM, pages 166-170, 2011. 
[50] M. Thorup. Simple tabulation, fast expanders, double tabulation, and high independence. In Proc. 54th IEEE Symposium on Foundations of Computer Science (FOCS), pages 90-99, 2013.

[51] M. Thorup and Y. Zhang. Tabulation-based 5-independent hashing with applications to linear probing and second moment estimation. SIAM Journal on Computing, 41(2):293-331, 2012. Announced at SODA'04 and ALENEX'10.

[52] M. N. Wegman and L. Carter. New classes and applications of hash functions. Journal of Computer and System Sciences, 22(3):265-279, 1981. Announced at FOCS'79.

[53] A. L. Zobrist. A new hashing method with application for game playing. Technical Report 88, Computer Sciences Department, University of Wisconsin, Madison, Wisconsin, 1970.

\section{A Conditioning on the hash value of a query key}

We are here going to argue that the Chernoff-style bounds from Theorem 5 hold when we for a given query key $q$ and hash value $a$ condition everything on $h(q)=a$. This will be a simple extension of the proof of Theorem 5 in $[42, \S 3]$ without the condition. Below we first describe the essential points from the proof in $[42, \S 3]$, referring the reader to $[42, \S 3]$ for more details. Afterwards we describe the extension to handle the condition.

No conditioning. In $[42, \S 3]$, the twisted tabulation hash function is picked in 3 steps.

1. We randomly fix $h^{\mathcal{T}}$ twisting the keys. This defines the twisted groups $G_{\alpha}$ consisting of keys with the same twisted head $\alpha$.

2. We randomly fix the $h_{i}^{\mathcal{S}}, i>0$, hashing the tails of the keys.

3. We randomly fix $h_{0}^{\mathcal{S}}$, hashing the twisted heads. This finalizes the twisted tabulation hashing $h=$ $h^{\mathcal{S}} \circ h^{\mathcal{T}}$.

Let $V_{\alpha}=\sum_{x \in G_{\alpha}} v_{x}(h(x))$ be the total value of keys in twisted group $G_{\alpha}$. The main technical point in [42, $\S 3]$ is to prove that, w.h.p., after step 2, we will have $V_{\alpha} \leq d=O(1)$ no matter how we fix $h_{0}^{\mathcal{S}}[\alpha]$, and this holds simultaneously for all twisted groups. While $h_{0}^{\mathcal{S}}[\alpha]$ has not been fixed, $h(x)$ is uniform in $[u]$ for every $x \in G_{\alpha}$, so the expected value $\mu_{\alpha}=\mathrm{E}\left[V_{\alpha}\right]=\sum_{x \in G_{\alpha}} \mu_{x}$ over $G_{\alpha}$ is unchanged before step 3. It also follows from [42, §3] that $\mu_{\alpha} \leq d$. Our final value is $V=\sum_{\alpha \in \Sigma} V_{\alpha}$ which still has the correct mean $\mathrm{E}[V]=\mu$. Moreover, $V$ is Chernoff-concentrated since it is summing $d$-bounded variables $V_{\alpha}$ as we fix the $h_{0}^{\mathcal{S}}[\alpha]$ in step 3 . We are here ignoring some technicalities explained in [41, 42], e.g., how to formally handle if some variable $V_{\alpha}$ is not $d$-bounded.

Conditioning on $\boldsymbol{h}(\boldsymbol{q})=\boldsymbol{a}$. We now consider the effects of the condition $h(q)=a$. First we note that since twisted tabulation is 2-independent, the condition $h(q)=a$ does not affect the expected value $\mu_{x}=\mathrm{E}\left[v_{x}(h(x))\right]$ of any key $x \neq q$.

Now, for the above step-wise fixing of the twisted tabulation hash function, we note that the only effect of the condition $h(q)=a$ is in step 3. If $\alpha_{0}$ is the twisted head of the query key, then instead of picking $h_{0}^{\mathcal{S}}\left[\alpha_{0}\right]$ at random, we have to fix it as $h_{0}^{\mathcal{S}}\left[\alpha_{0}\right]=a \oplus \bigoplus_{i=1}^{c-1} h_{i}^{\mathcal{S}}\left[q_{i}\right]$. Since the first steps were not affected, 
w.h.p, for all $\alpha \in \Sigma$, we still have that $V_{\alpha} \leq d=O(1)$ no matter how we fix $h_{0}^{\mathcal{S}}[\alpha]$, and this includes the twisted head $\alpha_{0}$.

We are now again considering $V=\sum_{\alpha \in \Sigma} V_{\alpha}$ where each $V_{\alpha}$ is $d$-bounded. For all $\alpha \neq \alpha_{0}$, we have $\mathrm{E}\left[V_{\alpha}\right]=\mu_{\alpha}$. However, $V_{\alpha_{0}}$ is fixed to some value in $[0, d]$ when we after steps 1-2 are forced to set $h_{0}^{\mathcal{S}}\left[\alpha_{0}\right]=a \oplus \bigoplus_{i=1}^{c-1} h_{i}^{\mathcal{S}}\left[q_{i}\right]$. The error $\left|V_{\alpha_{0}}-\mu_{\alpha_{0}}\right|$ from this fixing is less than $d$, and this has no effect on our error probability bounds except in cases where Markov's bound takes over. However, Markov's bound holds directly for twisted tabulation without any of the above analysis. Thus we conclude that Theorem 5 holds also when we condition on $h(q)=a$. 